\title{
Correction to: Phosphorylation of pericyte FAK-Y861 affects tumour cell apoptosis and tumour blood vessel regression
}

\author{
Delphine M. Lees ${ }^{1} \cdot$ Louise E. Reynolds $^{1}\left[\right.$ (1) $\cdot$ Ana Rita Pedrosa ${ }^{1} \cdot$ Marina Roy-Luzarraga ${ }^{1} \cdot$ Kairbaan M. Hodivala-Dilke $^{1}$
}

Published online: 4 July 2021

(c) The Author(s) 2021

\section{Correction to: Angiogenesis}

https://doi.org/10.1007/s10456-021-09776-8

In the original publication, Fig. 1 and Fig. 4 were published incorrectly. The correct figures are provided in this correction.

The original article has been corrected.

Open Access This article is licensed under a Creative Commons Attribution 4.0 International License, which permits use, sharing, adaptation, distribution and reproduction in any medium or format, as long as you give appropriate credit to the original author(s) and the source, provide a link to the Creative Commons licence, and indicate if changes were made. The images or other third party material in this article are included in the article's Creative Commons licence, unless indicated otherwise in a credit line to the material. If material is not included in the article's Creative Commons licence and your intended use is not permitted by statutory regulation or exceeds the permitted use, you will need to obtain permission directly from the copyright holder. To view a copy of this licence, visit http://creativecommons. org/licenses/by/4.0/.

The original article can be found online at https://doi.org/10.1007/ s10456-021-09776-8.

Kairbaan M. Hodivala-Dilke

k.hodivala-dilke@qmul.ac.uk

1 Adhesion and Angiogenesis Laboratory, Centre for Tumour Microenvironment, Barts Cancer Institute - a CR-UK Centre of Excellence, Queen Mary University of London, John Vane Science Centre, Charterhouse Square, London EC1M 6BQ, UK 
a

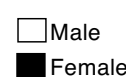

b

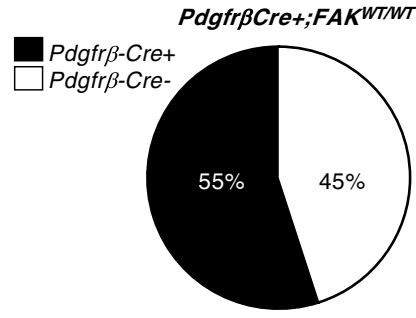

C
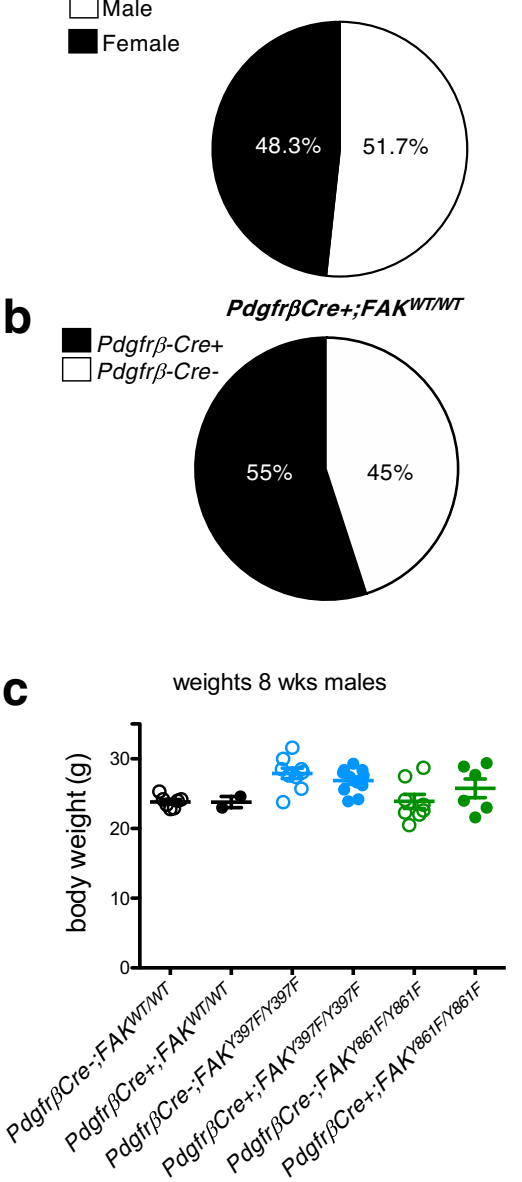

Pdgfr 3 Cre+;FAK ${ }^{\text {Y397F/Y397F }}$

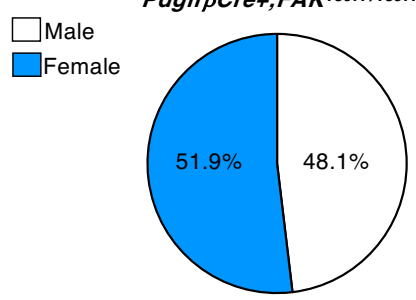

Pdgfr BCre+;FAK Y397F/Y397F

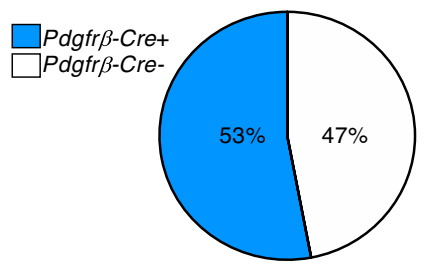

weights 8 wks females

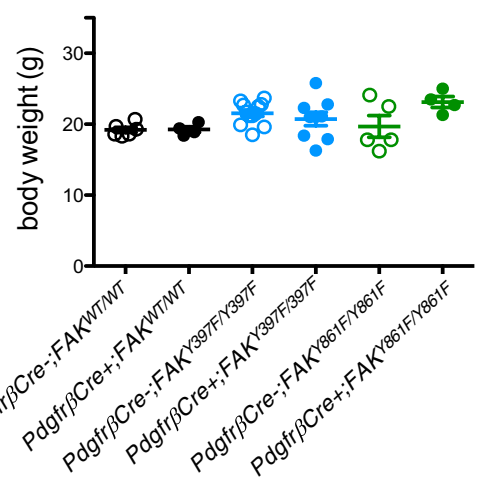

PdgfrßCre+;FAK ${ }^{Y 861 F / Y 861 F}$

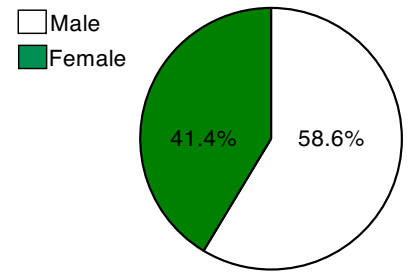

Pdgfr $\beta C r e+; F A K^{\text {Y861F/Y861F }}$

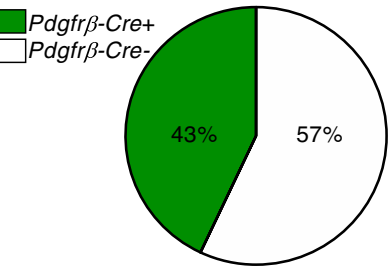

d pY861-FAK FAK WT-PC Y861F-PC e
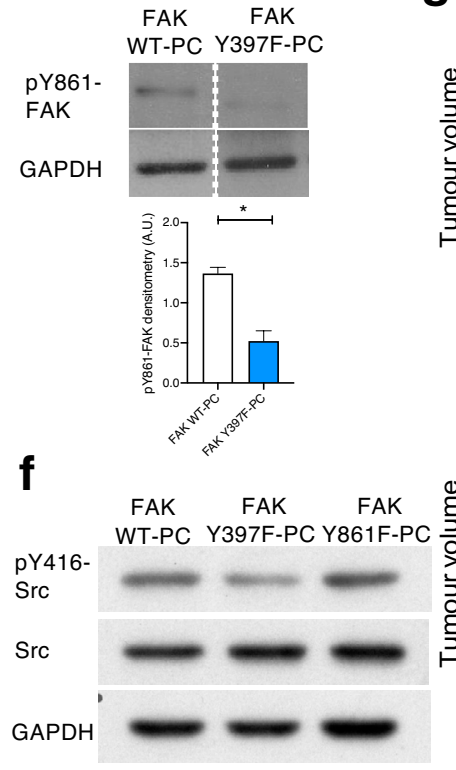

h
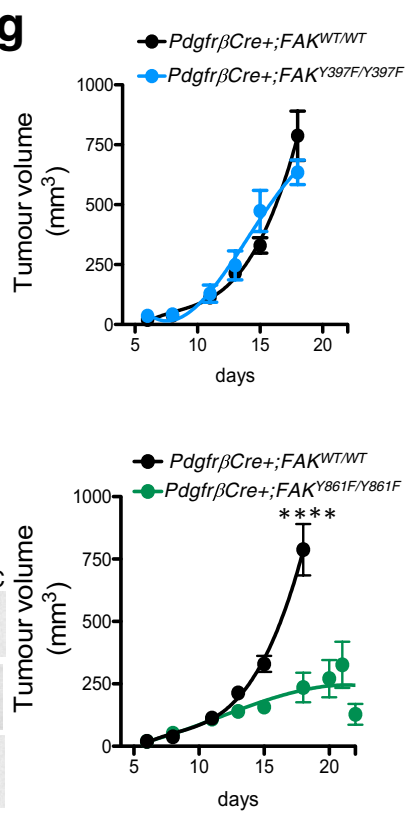
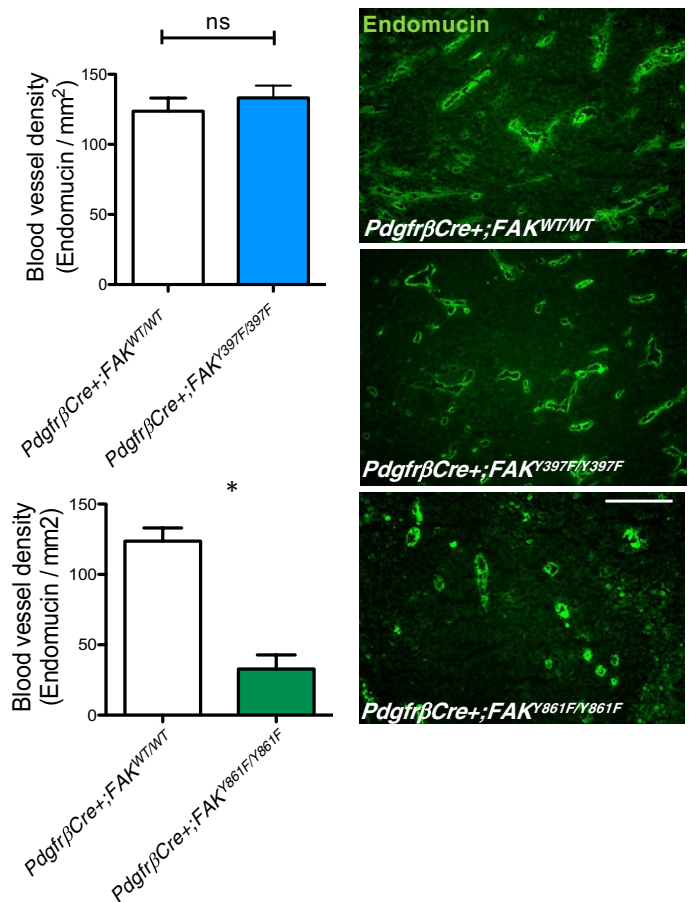
४Fig. 1 LLC tumour growth and angiogenesis are reduced in $\mathrm{Pdgfr} \beta \mathrm{Cre}+; \mathrm{FAK}^{\mathrm{Y} 861 \mathrm{~F} / \mathrm{Y} 861 \mathrm{~F}}$ mice. a Pdgfr $\beta C r e+; F A K^{W T / W T}, \quad P d g f r \beta C r e+; F A K^{Y 397 F / Y 397 F} \quad$ and Pdgfr $\beta C r e+; F A K^{Y 861 F / Y 861 F}$ mice were born at normal male;female ratios; b Mendelian ratios with $\mathbf{c}$ similar body weights. Pie chart in a represents percentage male:female ratio, in b represents \% Cre + and Cre- mice born to each genotype ( $n=60$ mice/ genotype). d Western blotting of primary pericytes isolated from Pdgfr $\beta C r e+; F A K^{W T / W T}, \quad P d g f r \beta C r e+; F A K^{Y 397 F / Y 397 F}$ and

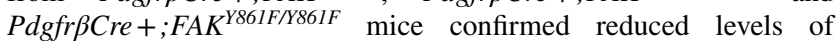
p-Y397 and pY861-FAK in FAKY397F and FAKY861F pericytes, respectively. e pY861-FAK levels are significantly reduced in Y397F pericytes, $n=2$ independent lysates/genotype. Bar chart represents mean pY861-FAK levels $+\mathrm{sd}$. $* P=0.0155$. GAPDH act as loading control. f pY416-Src levels are significantly reduced in Y397FFAK pericytes. Blots shows pY416-Src, total Src and GAPDH loading control. $\mathbf{g}$ In vivo tumour growth was significantly reduced only in Pdgfr $\beta C r e+; F A K^{Y 861 F / Y 861 F}$ mice. Graphs represent mean tumour volume \pm s.e.m.; $n=15$ Pdgfr $\beta C r e+; F A K^{W T / W T}$ mice, 14 Pdgfr $\beta C r e+; F A K^{Y 397 F / Y 397 F}$ mice and 11 Pdgfr $\beta C r e+; F A K^{Y 861 F / Y 861 F}$ mice. $* * * * P<0.0001$. Two-sided Mann-Whitney $U$ rank sum test. h Tumour blood vessel density was significantly reduced only in Pdgfr $\beta C r e+; F A K^{Y 861 F / Y 861 F}$ mice. Bar charts represent mean blood vessel density + s.e.m. $* P=0.0498 ; \quad$ ns, not significant; $n=6$ Pdgfr $\beta C r e+; F A K^{W T / W T}$ tumours, 6 Pdgfr $\beta C r e+; F A K^{Y 397 F / Y 397 F}$ tumours and 5 Pdgfr $\beta C r e+; F A K^{Y 861 F / Y 861 F}$ tumours. Two-sided Student's $t$-test. Representative endomucin stained LLC tumour sections are shown for each genotype. Scale bar, $50 \mu \mathrm{m}$ 

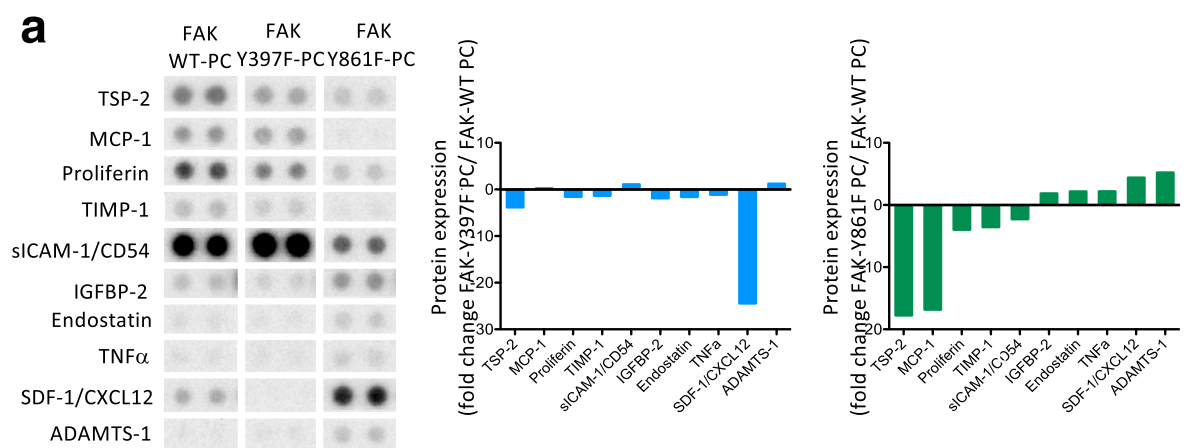

b
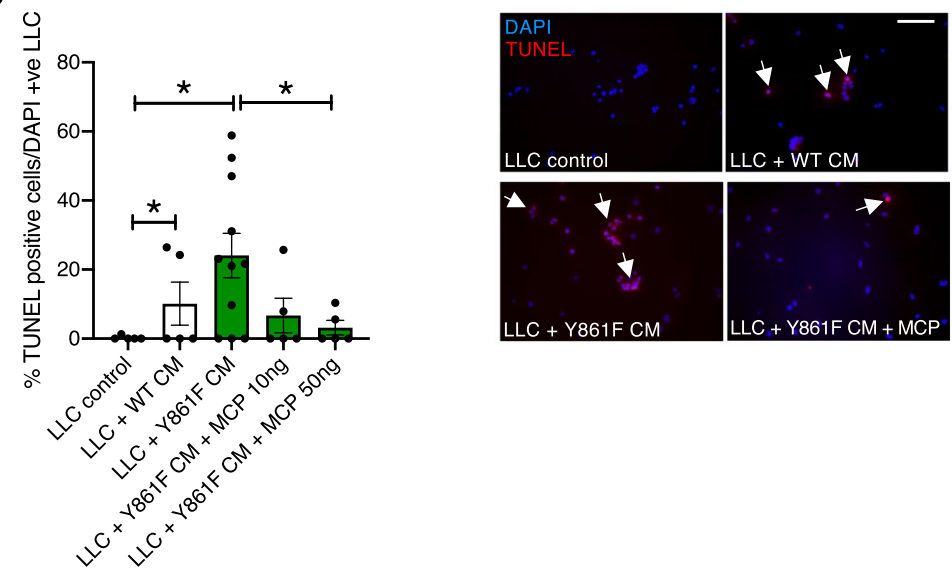

C
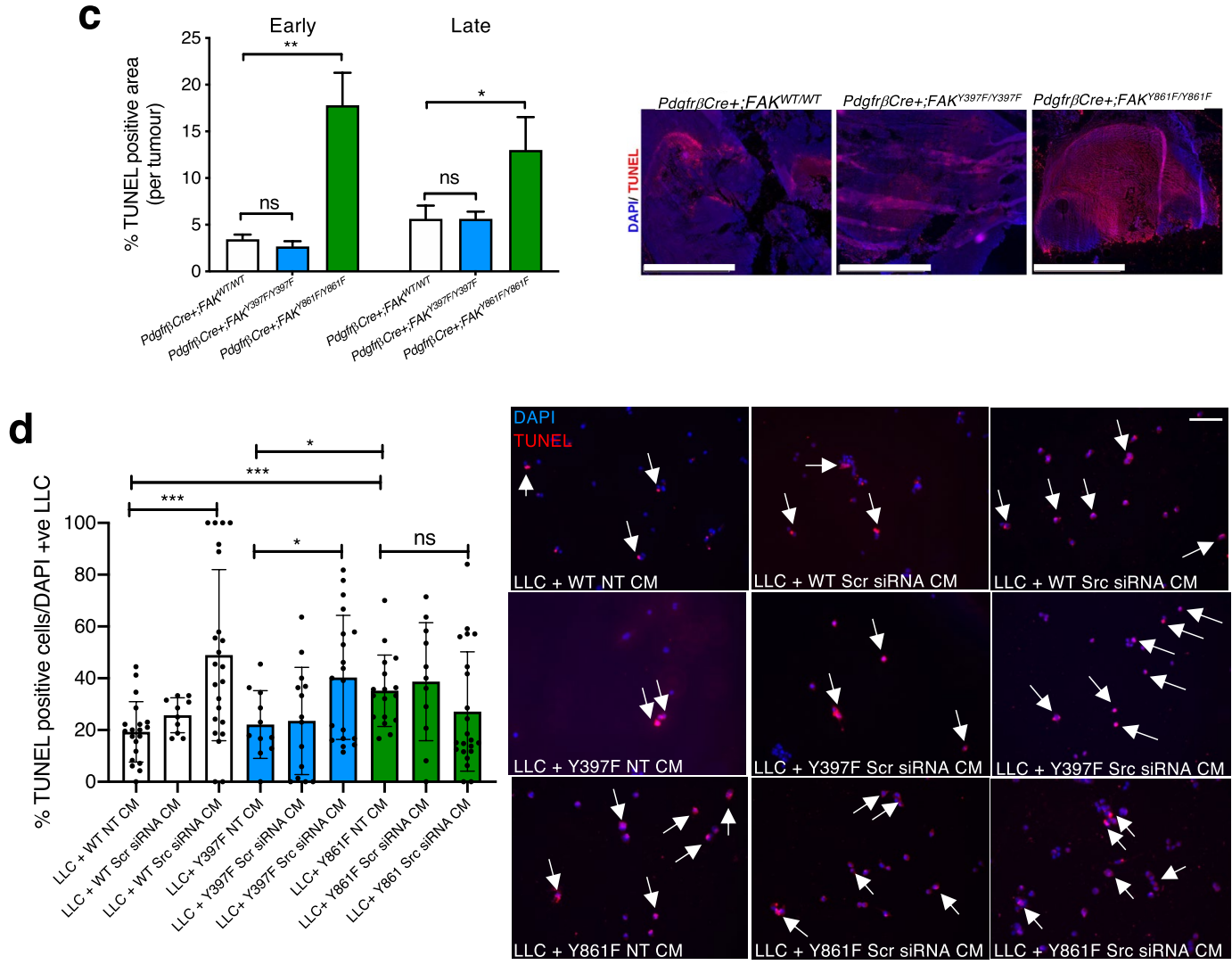
4Fig. 4 FAK-Y861F pericytes induce apoptosis in LLC tumour cells. a R\&D proteome profiler array using lysates from FAK-WT, FAK-Y397F and FAK-Y861F pericytes. Representative dots of differentially expressed proteins are given. Bar charts show mean fold change in protein expression relative to levels in WT. $N=2$ dots from 1 experiment. b Lewis lung carcinoma (LLC) cells incubated with conditioned medium $(\mathrm{CM})$ from pericytes plus or minus recombinant MCP-1. Treatment with FAK-Y861F pericyte CM increased LLC apoptosis, compared with CM from FAK-WT pericytes. This effect was rescued upon treatment with MCP-1 (10 and $50 \mathrm{ng} / \mathrm{ml}$ ). Bar chart represents \% TUNEL-positive cells + s.e.m. Representative images show effect of $\mathrm{CM}$ and MCP-1 on LLC cells. Arrows, TUNEL-positive cells. $* P<0.05$. One-way ANOVA. $N=5-11$ fields of view/genotype. Scale bar, $500 \mu \mathrm{m}$. c Early and late stage tumours from Pdgfr $\beta C r e+; F A K^{Y 861 F / Y 861 F}$ mice had significantly larger TUNEL-positive areas than tumours from Pdgfr $\beta C r e+; F A K^{W T / W T}$ and Pdgfr $\beta C r e+; F A K^{Y 397 F / Y 397 F}$ mice. Bar chart shows $\%$ TUNEL-positive area/tumour + s.e.m. $* * P=0.0012$, $* P=0.0464 ;$ ns, not significant; $n=6$ Pdgfr $\beta C r e+; F A K^{W T / W T}$ mice, 8 Pdgfr $\beta C r e+; F A K^{Y 397 F / Y 397 F}$ mice and 6 Pdgfr $\beta C r e+; F A K^{Y 861 F / Y 861 F}$ mice. Two-way ANOVA. Representative images show TUNEL-positive staining in tumours. Scale bar, $2.5 \mathrm{~mm}$. d LLC cells incubated with CM from pericytes treated with Src siRNA, non-specific scrambled siRNA (Scr) or transfection reagent (NT). CM from non-treated (NT) Y861F had a significantly higher effect on LLC apoptosis compared with CM from either WT or Y397F pericytes. Knockdown of Src in both WT and Y397F pericytes significantly increased LLC apoptosis. Src knockdown in Y861F pericytes did not increase LLC apoptosis above control levels. Bar chart represents \% TUNEL-positive cells \pm s.e.m. $* * * P=0.0006, * P=0.0196$ One -way ANOVA. $* * * P=0.0006$ WT NT vs. $861 \mathrm{~F} \mathrm{NT,} * P=0.03$ (Y397F NT vs. Y861F NT). Two-sided students $t$ test. $N=10-23$ fields of view. Scale bar, $500 \mu \mathrm{m}$; arrows in $\mathbf{b}$ and $\mathbf{d}$ indicate TUNEL-positive cells
Publisher's Note Springer Nature remains neutral with regard to jurisdictional claims in published maps and institutional affiliations. 